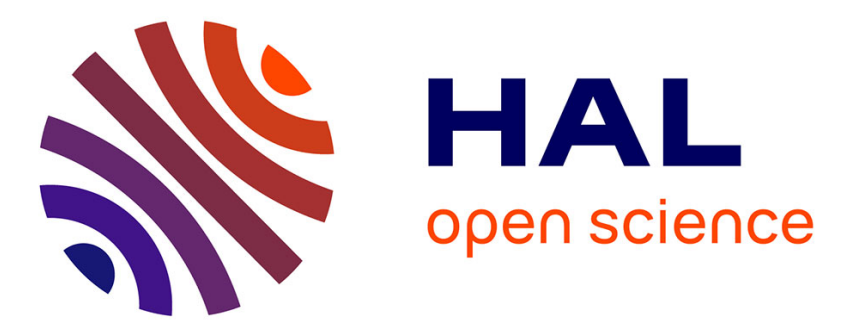

\title{
Pore-scale numerical simulation of the thermal performance for phase change material embedded in metal foam with cubic periodic cell structure \\ Xusheng $\mathrm{Hu}$, Xiao-Lu Gong
}

\section{- To cite this version:}

Xusheng $\mathrm{Hu}$, Xiao-Lu Gong. Pore-scale numerical simulation of the thermal performance for phase change material embedded in metal foam with cubic periodic cell structure. Applied Thermal Engineering, 2019, 151, pp.231-239. 10.1016/j.applthermaleng.2019.02.004 • hal-02281516v2

\section{HAL Id: hal-02281516 \\ https://hal-utt.archives-ouvertes.fr/hal-02281516v2}

Submitted on 21 Dec 2021

HAL is a multi-disciplinary open access archive for the deposit and dissemination of scientific research documents, whether they are published or not. The documents may come from teaching and research institutions in France or abroad, or from public or private research centers.
L'archive ouverte pluridisciplinaire HAL, est destinée au dépôt et à la diffusion de documents scientifiques de niveau recherche, publiés ou non, émanant des établissements d'enseignement et de recherche français ou étrangers, des laboratoires publics ou privés.

\section{(ㄷ)(1) $\$$}

Distributed under a Creative Commons Attribution - NonCommerciall 4.0 International 


\section{H-Bond Catalytic Mechanism of Aromatic Electrophilic Substitution between Phenol and Formaldehyde}

Jie Zhang ${ }^{\mathrm{a}^{*}}$, Tong Zhao ${ }^{\mathrm{b}}$, Ju Xie $\mathrm{e}^{\mathrm{c}^{*}}$, Zedong He ${ }^{\mathrm{a}}$, William W. Yu ${ }^{\mathrm{a}}$

${ }^{a}$ Key Laboratory of Special Functional Aggregated Materials and Key Laboratory of Colloid and Interface Chemistry, Ministry of Education, School of Chemistry and Chemical Engineering, Shandong University, Jinan 250100, China

${ }^{\mathrm{b}}$ Laboratory of Advanced Polymer Materials, Institute of Chemistry, Chinese Academy of Sciences, Beijing 100190, China

c School of chemistry and chemical engineering, Yangzhou University, Yangzhou 225002, China

* Corresponding author. E-mail: jiezhang@sdu.edu.cn (Jie Zhang), xieju@yzu.edu.cn (Ju $\mathrm{Xie})$ 


\begin{abstract}
Phenolic resins are widely used as moulding plastics, coatings, industrial bondings and ablative materials. An essential reaction step for preparing phenolic resins is aromatic electrophilic substitution. However, the current understanding of this reaction mechanism is kind of too general, making them not able to explain some phenomena such as highly exothermic characteristic of the early reaction stage between phenol and aldehyde under acid catalysis, and how attacking species form when the reaction begins. We studied the detailed catalytic mechanism by quantum mechanics. A new hydrogen-bond (H-bond) catalytic mechanism is suggested. It reveals that the essence of the acid catalytic mechanism is electrophile activation through hydrogen-bonding, and the substrate-catalyst complex formed through an H-bond is the attacking species. Besides, the study discloses that oxalate anion takes part in the reaction through bonding with arenium ion. The thermodynamic and kinetic characters based on this mechanism are quite different from the current theories.
\end{abstract}

Keywords: H-Bond Catalytic Mechanism; Aromatic Electrophilic Substitution; Computational chemistry 


\section{Introduction}

Phenolic resins have broad applications such as moulding plastics, coatings, industrial bondings and ablative materials. ${ }^{1,2}$ Thermoplastic phenolic resins are synthesized by the reaction of phenol with formaldehyde in an acidic medium. It is a strong exothermic reaction at the beginning of the process. ${ }^{3}$ The large amount of heat is thought to result from the aromatic electrophilic substitution, which is usually called addition-elimination process as shown in Figure 1. However, there is no reasonable explanation for the strong exothermic phenomenon based on the mostly accepted mechanism. According to this mechanism, an arenium ion firstly forms in an endothermic step, which is thought to be the rate determining step. Next is deprotonation, and it is faster than the first one. ${ }^{4}$ There is no sign that a lot of heat is released in this step. In spite of its broad acceptance, the essential reaction to produce phenolic resin has not been sufficiently studied.

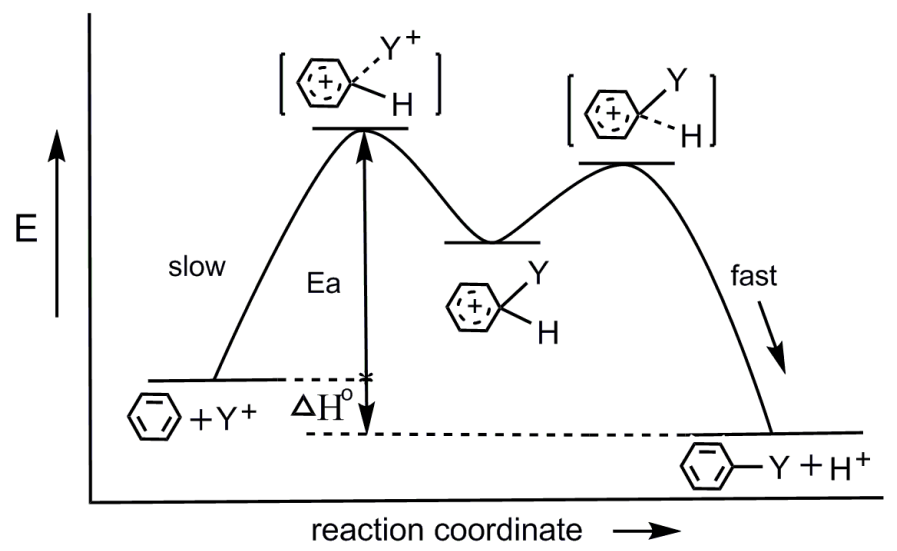

Figure 1. Potential energy diagram of the classical mechanism of aromatic electrophilic substitution.

Some experimental studies on phenolic resin preparation focused on the kinetics of substitution (namely addition) and condensation reactions in alkaline medium, including 
reaction rate constant and reaction order. ${ }^{5-7}$ However, the details for every step cannot be obtained by experimental methods.

Computational chemistry is especially important for elucidating mechanisms of chemical reactions. ${ }^{8-12}$ Aromatic electrophilic substitution is also one of the objects of computational chemistry. ${ }^{13-15}$ As a research mothod, it gives new thoughts on it. ${ }^{16-18}$ Hill and Conner studied the reactivity of phenolic compounds with formaldehyde under basic conditions. ${ }^{19,20}$ But computational studies on the reaction under acidic condition are very few. ${ }^{21,}{ }^{22}$ It is generally accepted that in the arenium ion mechanism the attacking species may form in various ways, but what happens on the aromatic ring is basically the same in all cases. For this reason, most mechanism studies mainly focus on the identity of the attacking entities and how they are formed. The acid-catalyzed mechanism has been investigated at the PW91/DNP level with COSMO (conductor-like screening model) solvent model. ${ }^{21}$ However, the data cannot explain the highly exothermic characteristic of the reaction, because protonated methanediol or protonated formaldehyde is treated as the attacking entities. Besides, the effect of the counterion of proton (i.e., the catalyst anion, or the conjugated base) has not been revealed in the acid catalyzed mechanism. A more detailed mechanism is needed to help us further understand the reaction.

From the beginning of this century, small-molecule chiral hydrogen-bond (H-bond) donors have been used to catalyze an array of C-C and C-heteroatom bond-forming reactions with high enantioselectivity and broad scope of substrates. ${ }^{23}$ The derivatives of thiourea ${ }^{24,25}$ and phosphoric acid ${ }^{26-28}$ are the most representative catalysts employed in H-bond catalyses. Experimental and theoretical studies of catalytic reactions reveal that $\mathrm{H}$-bonding interaction between catalysts and substrates is the key activation mechanism. For example, Jacobsen 
testified the formation of an imine-catalyst complex through an $\mathrm{H}$-bond between the imine nitrogen and an acidic proton of the urea catalyst in Strecker reaction. ${ }^{24}$ In their review article on small-molecule chiral H-bond donors, Doyle and Jacobsen pointed out that elucidation of fundamentally distinct modes of activation of Brønsted-acid catalysts would likely inspire the design of new $\mathrm{H}$-bond acid catalysts and bring in new reactions and reaction partners for enantioselective catalysis. ${ }^{23}$

In this paper, an H-bond catalytic mechanism for aromatic electrophilic substitution between phenol and formaldehyde in a Brønsted-acidic medium is presented. It discloses that the essence of the catalysis is electrophile activation through $\mathrm{H}$-bonding. Besides, the effect of the conjugate Brønsted base is also proposed. This mechanism illustrates that the aromatic electrophilic substitution is a highly exothermic reaction, which is well consistent with the experimental observation. ${ }^{3}$

\section{Methods}

Optimized geometries of all species and the stationary points on the potential surface were obtained at the MP2(fc)/6-31+G(d,p) level of theory. ${ }^{29-33}$ The single point energies of all optimized structures were obtained at the level of MP2(fc)/6-311++G(3df,2p) to increase the accuracy of the energy values. The solvent effect in water was modeled using the self-consistent reaction field (SCRF) method with the Tomasi's polarized continuum model (PCM). ${ }^{34,} 35$ The corresponding harmonic frequencies were calculated based on the MP2(fc)/6-31+G(d,p) optimized geometries to obtain zero-point energies (ZPEs) and to determine all stationary points as either minima (no imaginary frequencies) or transition states (only one imaginary frequency). The reaction paths were examined by intrinsic coordinate reaction (IRC) calculations at the MP2(fc)/6-31+G(d,p) level. The IRC results are reported in the Supporting Information. Based on the MP2(fc)/6-31+G(d,p) optimized 
geometries, natural bond orbital (NBO) analyses at the same level were then used. ${ }^{36}$ The Gaussian 09 series of programs were employed in all calculations. ${ }^{37}$

\section{Results and discussion}

\section{Formation of Attacking Specie: H-bonded complex}

For the aromatic electrophilic substitution, the most important thing is to determine the attacking species. Formaldehyde is usually used in aqueous solution. So in a previous computational study, methylene glycol was thought to be the existence form of formaldehyde in aqueous solution, then protonated methylene glycol and protonated formaldehyde were treated as the attacking entities considering an acid environment. ${ }^{21}$ But this leaves a few things not well explained. First, according to our calculations, the process from formaldehyde to methylene glycol has a barrier of $153.1 \mathrm{~kJ} / \mathrm{mol}$ though it is exothermic of $21.6 \mathrm{~kJ} / \mathrm{mol}$ (Figure S1). Second, it is well known that methylene glycol is thermodynamically unstable and is liable to revert to formaldehyde through dehydration under acidic conditions. ${ }^{4}$ Our calculations show that this dehydration reaction proceeds without a barrier (Scheme S1), which testifies that protonated methylene glycol is difficult to exist in an acidic system. If protonated formaldehyde is considered as the attacking entity, it should be formed first. However, the calculated results show that the formation of protonated formaldehyde is endothermic (Scheme S2). So, in terms of energy, both protonated methylene glycol and protonated formaldehyde are not the dominant attacking entities.
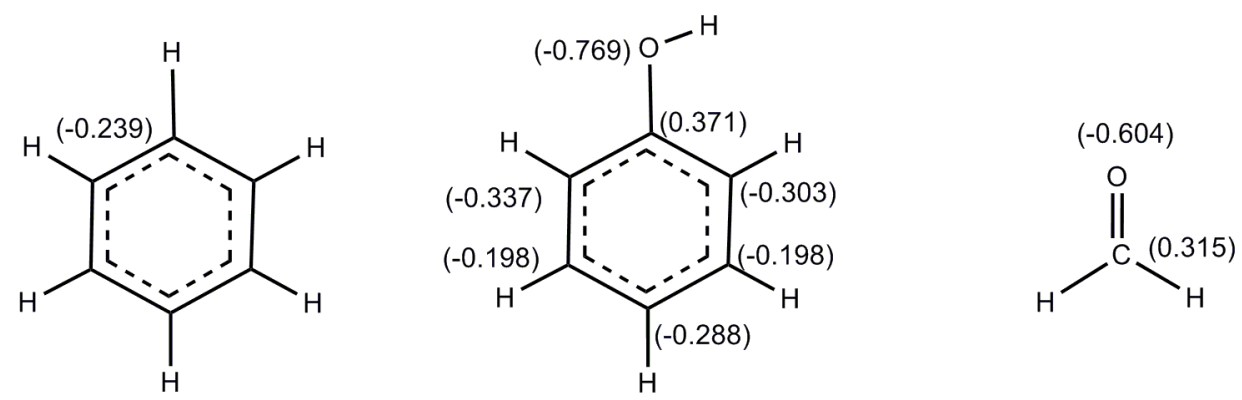
Figure 2. NBO charge distribution of benzene, phenol and formaldehyde at MP2(fc)/6-31+G(d,p) level.

The charge distributions can help to predict the initial state of reaction system and to judge the reactive site. Natural bond orbital (NBO) analysis was carried out and the NBO charges are shown in Figure 2. It can be seen that phenolic hydroxyl changes the charge distribution of the aromatic ring. The carbon atoms on ortho or para positions carry more negative charges than meta ones. Therefore, the ortho- and para-carbon atoms are more likely to be attacked by electrophilic reagents. ${ }^{38}$ Considering the high dissociation constant of oxalic acid $\left(\mathrm{pK}_{\mathrm{a} 1}=1.27\right)$ in aqueous, hydrated proton instead of oxalic acid molecule is involved in the reaction. The oxygen atoms of phenolic hydroxyl and carbonyl of formaldehyde as well as aromatic ring are all possible hydrated proton acceptors. Thus, three types of substrate-catalyst complexes through an H-bond have been optimized as shown in Scheme 1 as well as their binding energies.

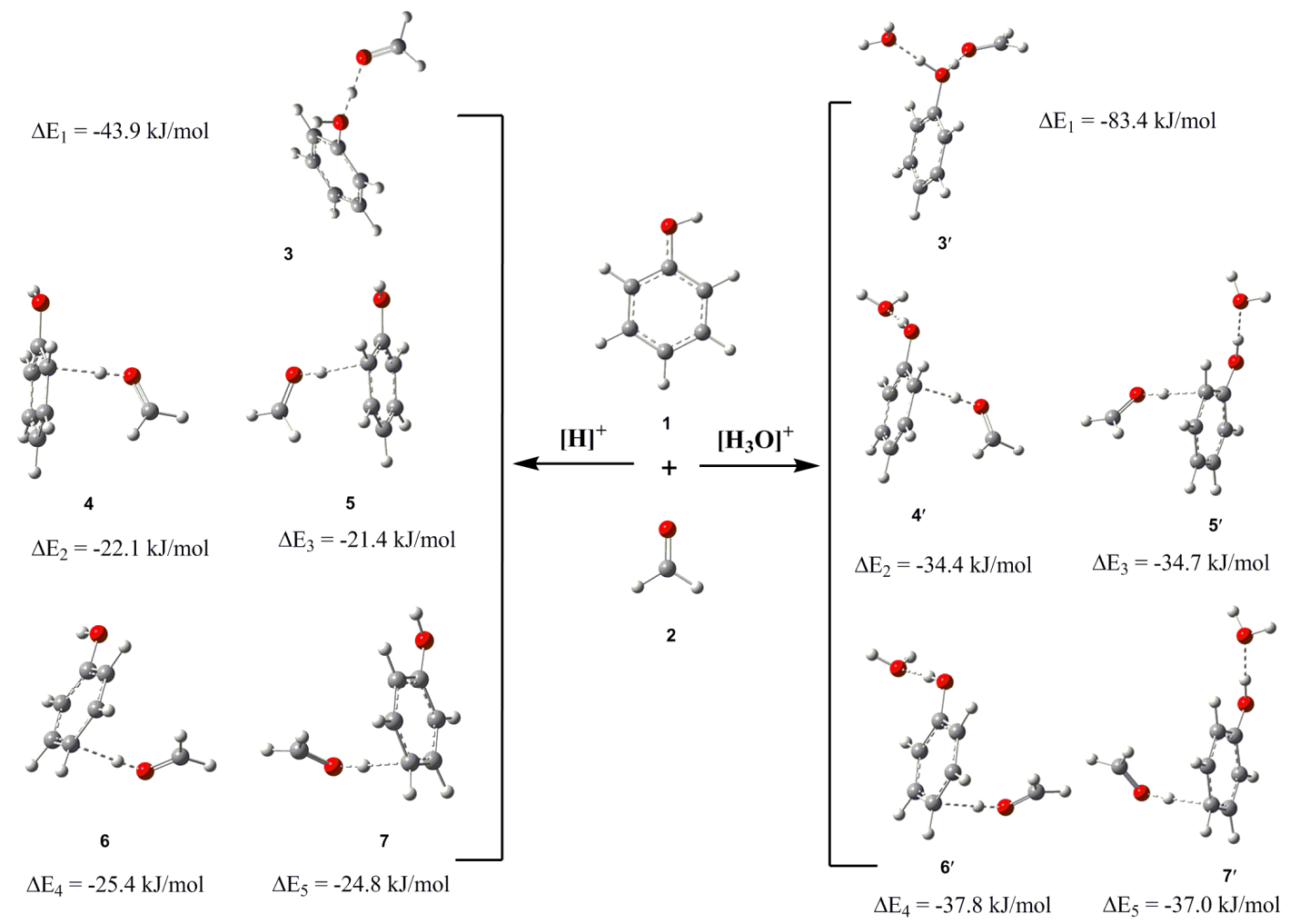


Scheme 1. The formation of H-bonded complexes, regarding proton only (left) and regarding hydrated proton (right).

It can be seen in Scheme 1 (right) that when $\mathrm{H}$-bonded complexes are formed, $\mathrm{H}_{2} \mathrm{O}$ molecule disassociates from the hydrated proton and forms an $\mathrm{H}$-bond with phenolic hydroxyl. This is the same in all five complexes. This type of H-bonding stabilizes the initial state of the reaction system. However, it is not the reactive site for electrophilic substitution. Therefore, $\mathrm{H}_{2} \mathrm{O}$ does not affect the acid catalytic mechanism much, but proton plays the major role. Proton can bridge phenol and formaldehyde to form another type of H-bond, which not only stabilizes the initial state of the reaction system, but also decides the next reaction.

With this consideration, we also optimized a simplified model of H-bonded complex regarding proton only (Scheme 1, left). It can be seen that the ways of formation of H-bond in reactive site are not affected by $\mathrm{H}_{2} \mathrm{O}$ molecule. The involvement of $\mathrm{H}_{2} \mathrm{O}$ molecule only influences the absolute values of the binding energy of the five H-bonded complexes, but it does not affect the relative magnitude of their stability.

Since there is no barrier to form H-bonded complexes, it can be confirmed that their formation is not the rate determining step for the multi-step aromatic electrophilic substitution.

As can be seen in Scheme 1, 3 is the most stable complex, which is formed by oxygen atom of phenolic hydroxyl, proton, and formaldehyde. The bridging interaction stabilizes $\mathbf{3}$ by $43.9 \mathrm{~kJ} / \mathrm{mol}$, which is stronger than the classical $\mathrm{O}-\mathrm{H} \cdots \mathrm{O} \mathrm{H}$-bond. ${ }^{24}$ The complexes $\mathbf{4 , 5}$, 6, and 7 use $\pi$ electrons on aromatic ring as electron donor at ortho or para positions. The $\mathrm{O}$ $-\mathrm{H} \cdots \pi \mathrm{H}$-bond leads to $21-25 \mathrm{~kJ} / \mathrm{mol}$ stabilization energy to the complexes, because $\mathrm{O}-$ 
$\mathrm{H} \cdots \pi$ is a relatively weak $\mathrm{H}$-bond. ${ }^{37}$

There are experimental evidences that a class of uncertain structure intermediates, named encounter complexes, are present before arenium ion is formed..$^{38}$ They are called as $\pi$ complexes. However, experimental results on $\pi$ complexes contradict each other. Some suggested that $\pi$ complex formation might be the rate determining step, ${ }^{39-41}$ while some suggested that arenium ion was formed in the rate determining step. ${ }^{42-46}$ Based on the above results, we supposed that the encounter complexes are attacking species, and are substrate-catalyst complexes through an H-bond. Their formation hence leads to a new mechanism for the aromatic electrophilic substitution.

\section{H-bond catalysis mechanism}

As mentioned above, H-bonded complex is formed between catalyst and substrates. The distribution of the frontier molecular orbitals (FMOs) on the reaction system can help to predict the reactivity in the next step.

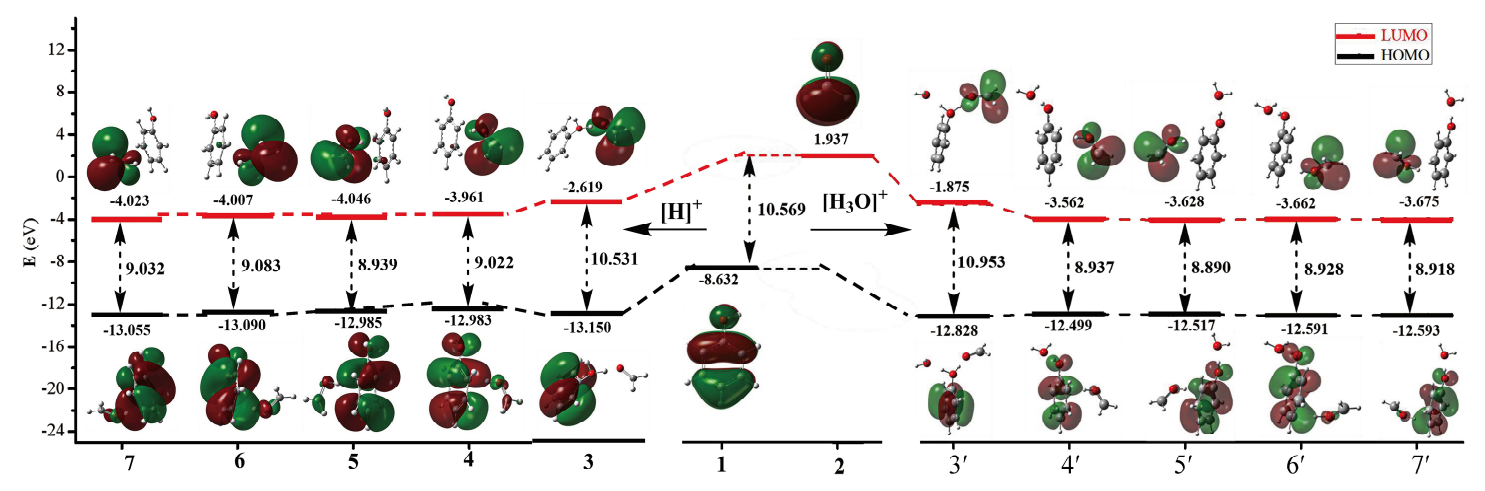

Figure 3. Frontier molecular orbitals of reactants and two models of H-bonded complex.

The FMOs of reactants (formaldehyde and phenol) and H-bonded complexes (3 7, 3' 7',) are shown in Figure 3. In order to further investigate the acid catalytic mechanism, FMOs of the two models of H-bonded complex were considered. From Fig.3 (right), it can be seen that 
there are no distributions of FMOs on $\mathrm{H}_{2} \mathrm{O}$ molecule which is released from hydrated proton. In other words, $\mathrm{H}_{2} \mathrm{O}$ molecule does not take part in the catalytic reaction. By now, it is reasonable to say that it is proton which plays the major role in catalytic action of oxalic acid. Therefore the simplified model of H-bonded complexes regarding proton only will be used as the attacking species and also for the sake of brevity, $\mathrm{H}_{2} \mathrm{O}$ molecule is not considered in the following sections.

It can be seen that in complex $\mathbf{3}$ the HOMO is located on the phenol fragment, while the LUMO is located on formaldehyde fragment. $\mathrm{O}-\mathrm{H} \cdots \mathrm{O}$ H-bond exists in this complex. In complexes $4, \mathbf{5}, \mathbf{6}$, and 7, the HOMO formed by using $\pi$ electrons on aromatic ring as electron donor mostly distributes on phenol, and a small part is situated on formaldehyde (on formaldehyde oxygen, specifically). The $\mathrm{O}-\mathrm{H} \cdots \pi \mathrm{H}$-bond is formed with formaldehyde oxygen, proton, and benzene ring in these complexes. In addition, the HOMO distribution on formaldehyde of the para-complexes $\mathbf{6}$ and $\mathbf{7}$ is slightly larger than that on the formaldehyde of ortho-complexes $\mathbf{4}$ and $\mathbf{5}$. It is worth mentioning that the formation of the H-bonded complexes significantly decreases the LUMO energy of the electrophilic formaldehyde and reduces the energy gap between the LUMO of formaldehyde and the HOMO of phenol. The energy gap between HOMO of phenol and LUMO of formaldehyde is $10.569 \mathrm{eV}$, while the energy gaps of the complexes are somewhat lower. For example, complex $\mathbf{5}$ has a decreased energy gap of $8.939 \mathrm{eV}$. This means the bridging H-bond interaction reduces the LUMO energy of the electrophile and enhances its electrophilic ability, thereby reduces the barrier of electrophilic substitution and catalyzes the reaction. This is consistent well with the typical LUMO activation mechanism of H-bond catalysis. ${ }^{24,47}$

The energy gap between LUMO and HOMO can be employed to predict the reactivity. A smaller energy gap represents a higher reactivity. Complexes $\mathbf{4 ,} \mathbf{5}, \mathbf{6}$, and $\mathbf{7}$ possess similar energy gap. The complex $\mathbf{3}$ formed by proton, formaldehyde and phenolic hydroxyl group 
has the highest energy gap. It can be predicted that the reaction starting with complex $\mathbf{3}$ has higher barrier than those of the reactions starting from other four complexes.

\section{From H-Bonded Complex to Arenium Ions}

\section{Ortho Arenium Ion}

$\mathbf{3}$ is the most stable complex. Starting from it, the ortho-arenium ion $\mathbf{1 0}$ is formed through three steps, as shown in Figure 4. The first step is the rate determining step with a barrier of $28.6 \mathrm{~kJ} / \mathrm{mol}$. Considering $43.9 \mathrm{~kJ} / \mathrm{mol}$ of heat that is released in the last step, the barrier is overcome easily. In this step, H-bond breaks and proton transfers to formaldehyde in transition state TS1, leading to the formation of intermediate $\mathbf{8}$. In the following two steps phenol and protonated formaldehyde get to close each other by adjusting their relative position via transition states TS2 and TS3 till ortho arenium ion $\mathbf{1 0}$ forms, in which C (phenol)-C (formaldehyde) bond is formed. There is almost no heat effect in the two steps, just endothermic by $0.8 \mathrm{~kJ} / \mathrm{mol}$. It can be seen that the ortho arenium ion $\mathbf{1 0}$ is more stable than reactants by $43.1 \mathrm{~kJ} / \mathrm{mol}$, although its aromaticity is destroyed. The covalent bonding and the delocalization of lone pair electrons of oxygen atom of phenolic hydroxyl to benzene ring contribute to its stability. 


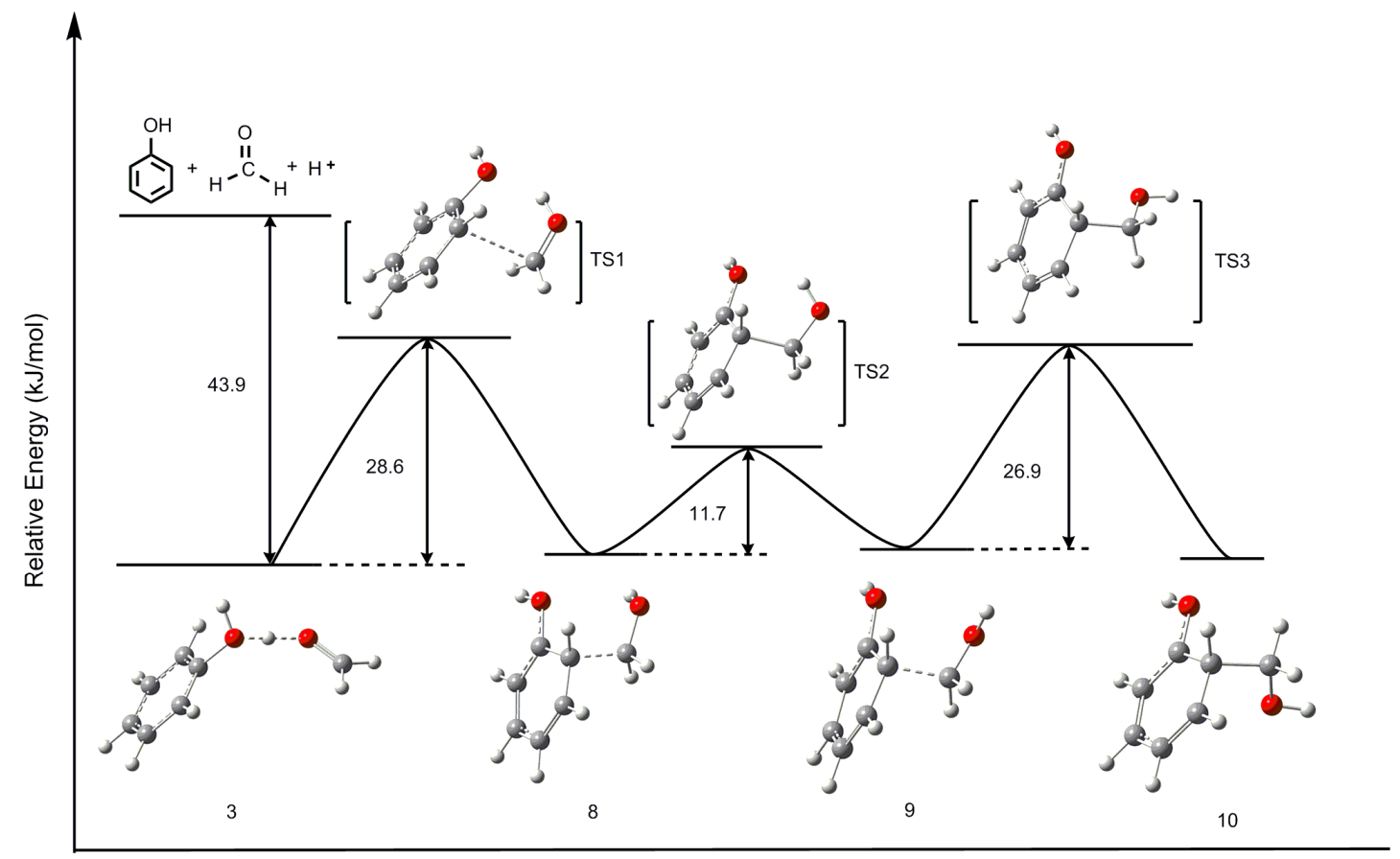

Figure 4. Energy profile of the formation of ortho arenium ion $\mathbf{1 0 .}$

Starting from complexes $\mathbf{6}$ and 7, ortho arenium ion $\mathbf{1 0}$ and $\mathbf{1 1}$ are formed after overcoming barriers of 8.6 and $9.2 \mathrm{~kJ} / \mathrm{mol}$, via transition states TS4 and TS5, respectively (Figure 5). In these two paths, para H-bonded complex with phenol, proton and formaldehyde first turns to ortho complex between phenol and protonated formaldehyde in transition state, and then $\mathrm{C}$ (phenol)-C (formaldehyde) bond is formed. The two paths are exothermic by 17.7 $\mathrm{kJ} / \mathrm{mol}$ and $14.0 \mathrm{~kJ} / \mathrm{mol}$, respectively. Overcoming a barrier of $18.8 \mathrm{~kJ} / \mathrm{mol}$, ortho arenium ion $\mathbf{1 1}$ turns to para arenium ion $\mathbf{1 2}$ via transition state TS6. 


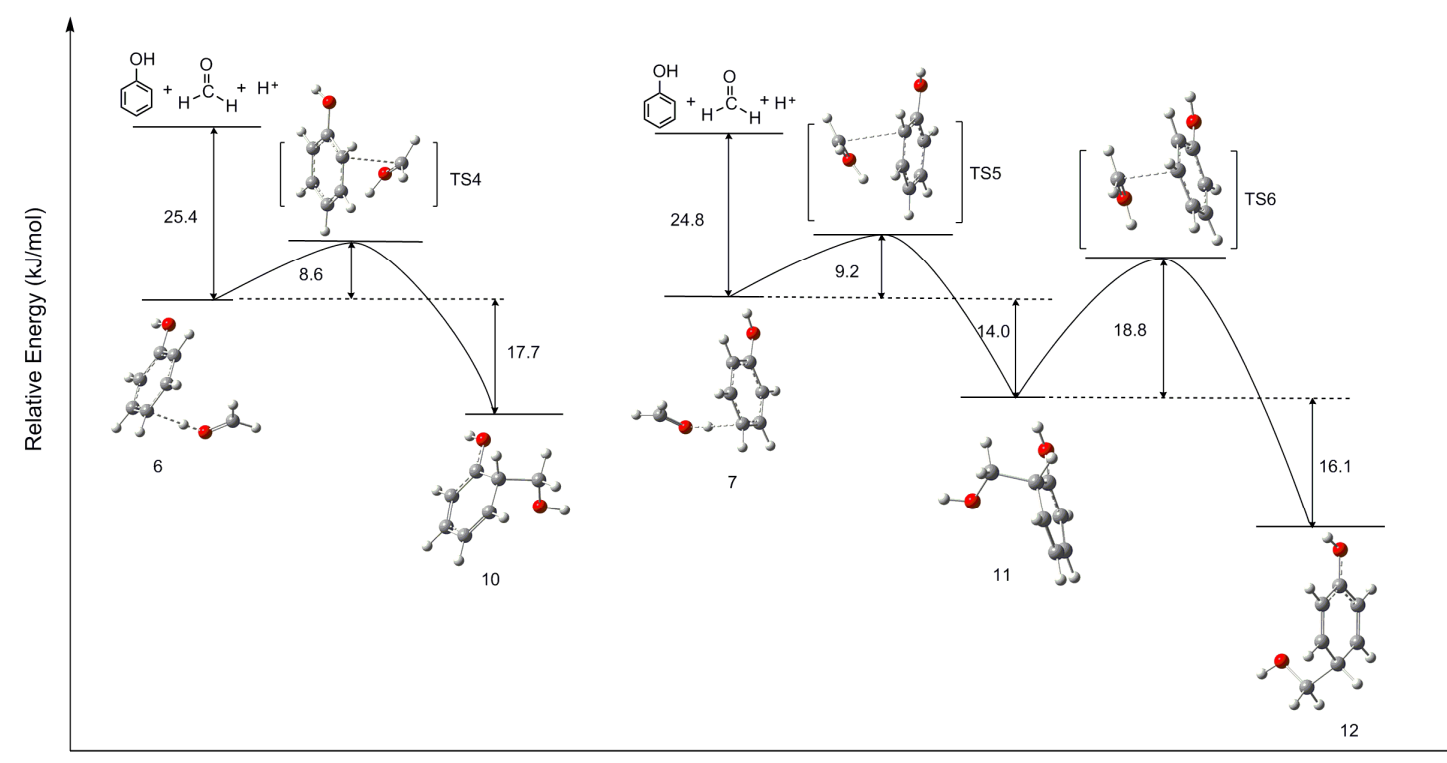

Figure 5. Energy profile of the formation of ortho arenium ions $\mathbf{1 0}$ and $\mathbf{1 1 .}$

Consider the reaction barriers, ortho arenium ion $\mathbf{1 0}$ is mainly formed from complex $\mathbf{6}$ rather than complex 3. Arenium ions 10, 11 and $\mathbf{1 2}$ are more stable than the corresponding reactants. The reason is given above.

\section{Para Arenium Ion}

Figure 6 shows that para arenium ion $\mathbf{1 2}$ is formed from complex $\mathbf{4}$ or complex $\mathbf{5}$. The two paths are exothermic by $32.9 \mathrm{~kJ} / \mathrm{mol}$ and $33.5 \mathrm{~kJ} / \mathrm{mol}$, respectively. Para arenium ion is also more stable than reactants, just like the ortho arenium ion. 


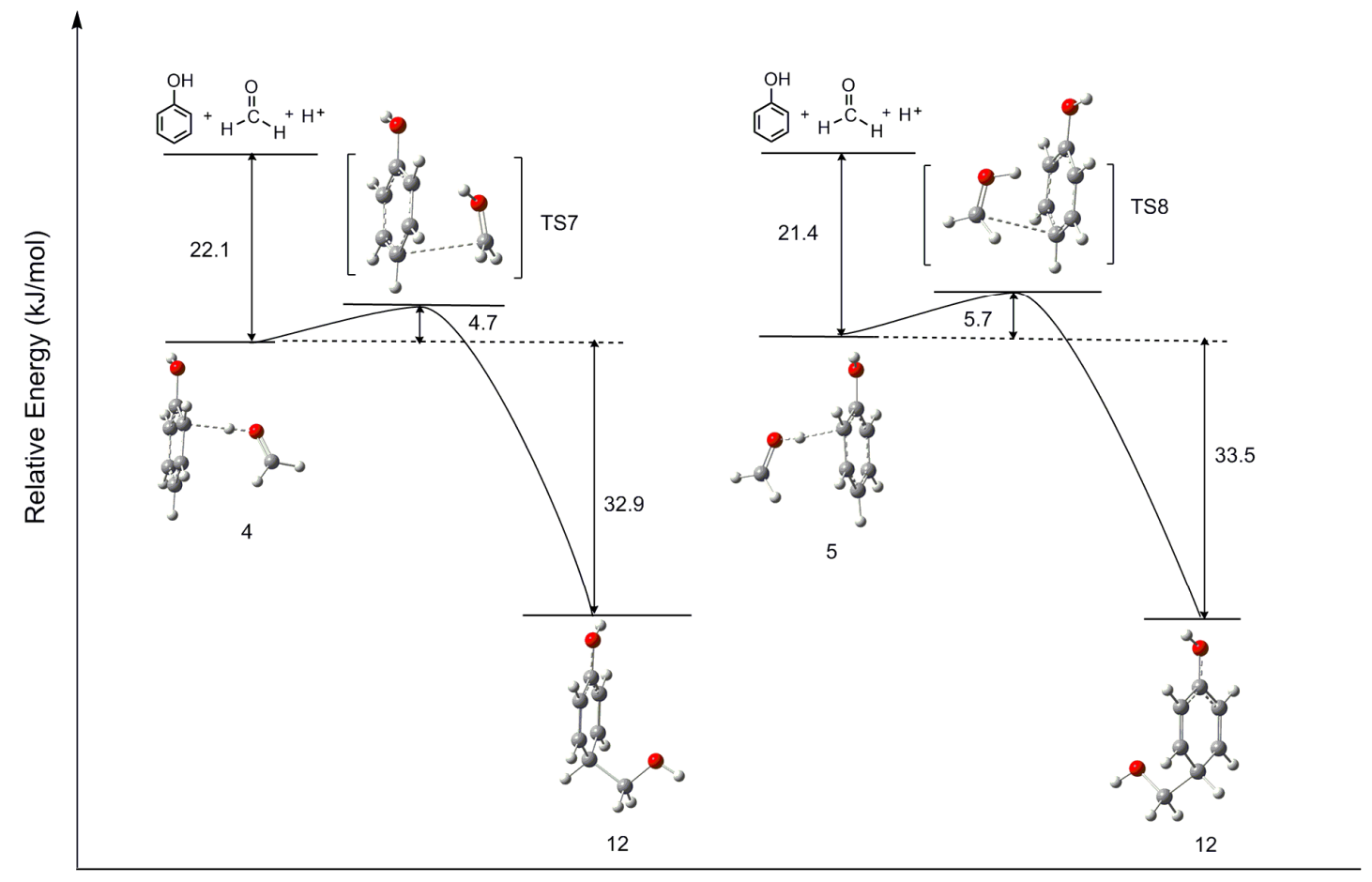

Figure 6. Energy profiles of the formations of para arenium ion 12.

In summary, the formation of arenium ion is an H-bond catalysis process. Oxalic acid decreases the reaction barrier through LUMO activation mechanism. Complexes 4, 5, 6 and 7 lead to paths with barriers not more than $10 \mathrm{~kJ} / \mathrm{mol}$, especially the paths from complexes 4 and 5 to para arenium ion 12 , only 4.7 or $5.7 \mathrm{~kJ} / \mathrm{mol}$. This means that the rate constants to form arenium ions are quite high. Once the four complexes are formed, they turn to arenium ions quickly. The path starting from complex 3 has a barrier of $28.6 \mathrm{~kJ} / \mathrm{mol}$, higher than those of other paths. The results testify our prediction on the reaction barriers based on the energy gaps between LUMO and HOMO of the complexes. The smaller the energy gap is, the lower the reaction barrier will be.

\section{The departure of Proton}

Following the formation of arenium ion is the loss of proton, and then a neutral substitution product is produced. It is certain that the proton departs with the help of a negative group. 
The ready negative group in reaction system is oxalate anion. We found that oxalate anion first combines with arenium ion through bonding the carbon adjacent to the site of electrophilic attack, because that carbon is electron-deficient, as natural charges shows in Figure 7 (numbers in blue). In addition, the adjacent position is favorable for the following hydrogen abstraction of oxalate anion.

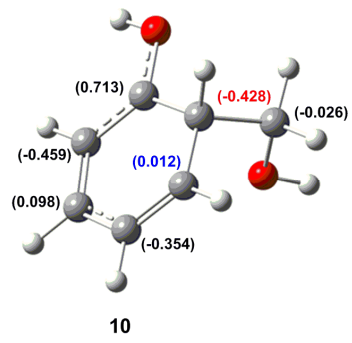

10

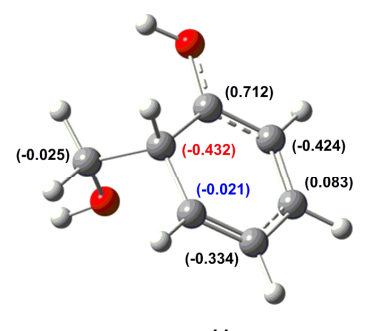

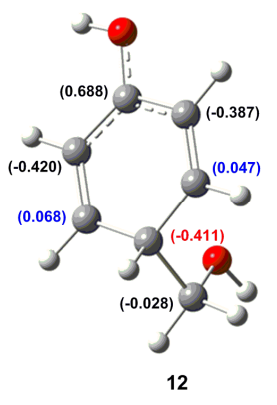

Figure 7. Natural charges (in parentheses) of arenium ions.

There are two ortho arenium ions, $\mathbf{1 0}$ and 11. For 10, as Figure 8 shows, it combines with oxalate anion to form a weak C-O bond (the bond length of C-O is $1.480 \AA$ ) and releases heat of $92.5 \mathrm{~kJ} / \mathrm{mol}$. Since it is a neutralization reaction between cation and anion, this step is exothermic and proceeds without reaction barrier. Then, the oxygen of the carbonyl of oxalate anion takes the proton from the adjacent carbon via transition state TS9, in which the C-O bond formed in the last step breaks. Thus, catalyst oxalic acid is released, and hydroxymethyl phenol $\mathbf{1 5}$ is formed. The aromatic electrophilic substitution at the ortho position to the phenol hydroxyl of phenol is finished. The heat of $195.4 \mathrm{~kJ} / \mathrm{mol}$ is released in the process. In the case of ortho arenium ion 11, the loss of proton is more complicated. It can be seen from Figure 9 that it goes through two steps and the reaction heat is $325.4 \mathrm{~kJ} / \mathrm{mol}$. 


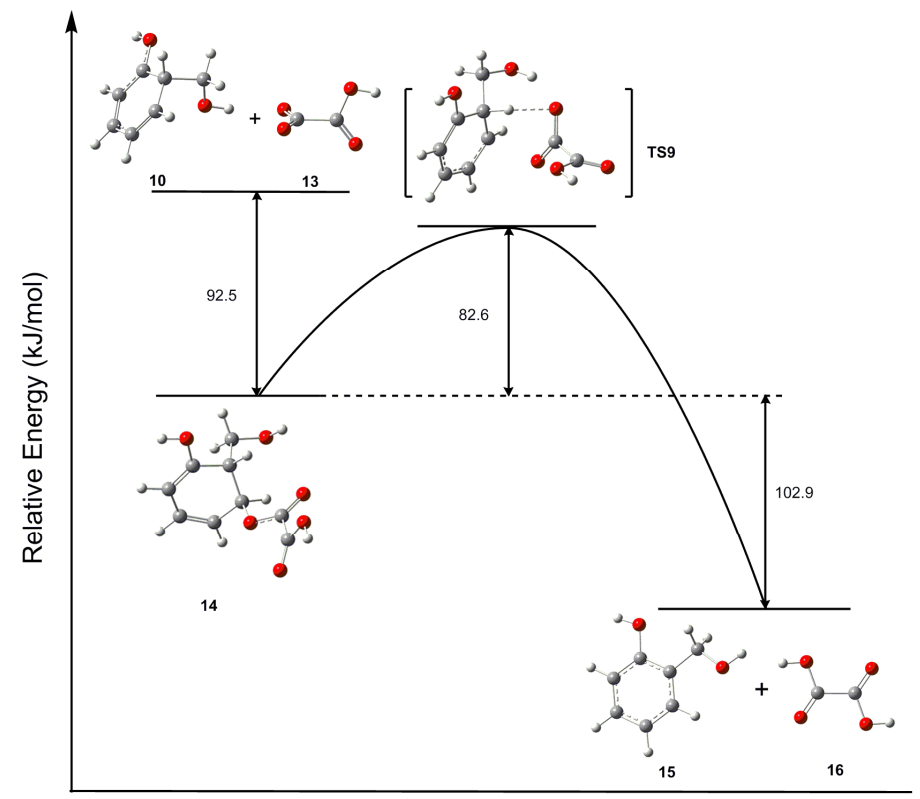

Figure 8. Energy profile of the formation of ortho substitute 15.

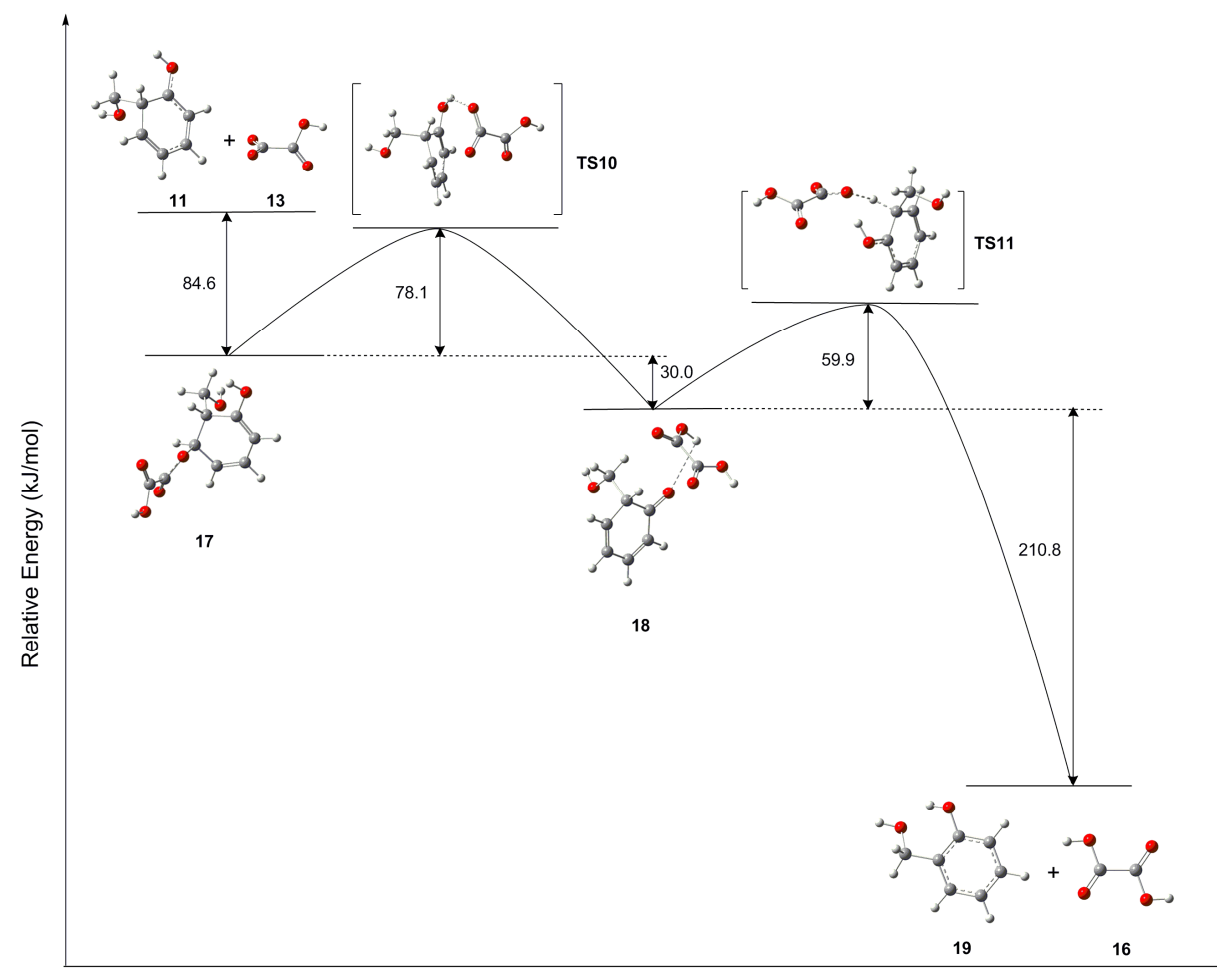

Figure 9. Energy profile of the formation of ortho substitute 19. 


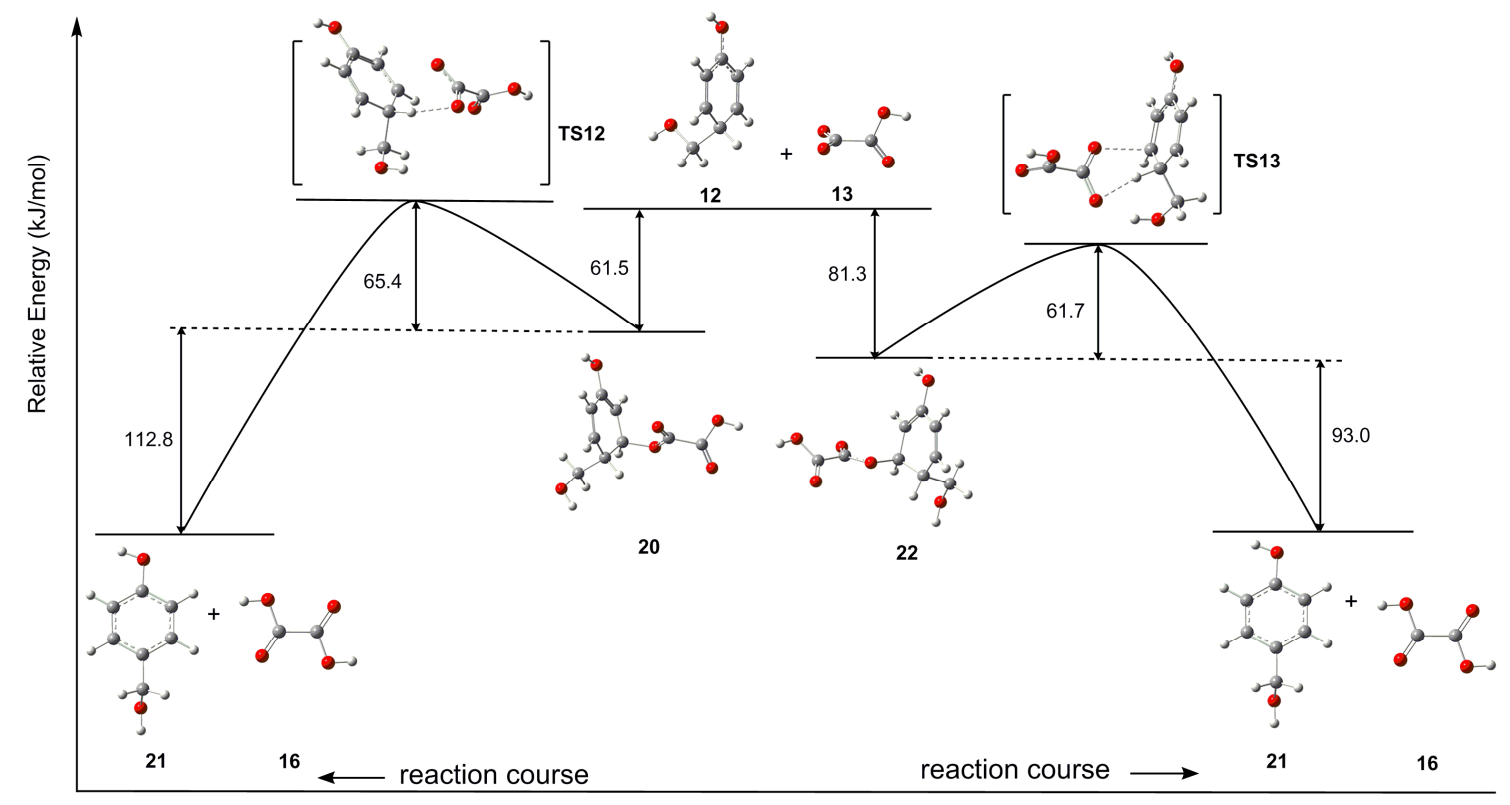

Figure 10. Energy profile of the formation of para substitute 21.

Para arenium ion $\mathbf{2 0}$ has two carbons adjacent to the site of electrophilic attack. So, there are two paths for the proton to leave. As Figure 10 shows, the reaction heats of two paths are 174.3 and $174.0 \mathrm{~kJ} / \mathrm{mol}$, respectively.

Figure 11 gives a summary of the whole reaction. It can be seen that every step, including the formation of H-bond complexes, the neutralization reaction between arenium ion and oxalate anion and the departure of proton, is exothermic. This means that the aromatic electrophilic substitution is a violent exothermal reaction, which is consistent with the experimental observation at the beginning stage to prepare phenolic resins in an acidic medium. ${ }^{3}$ The departure of proton contributes the most to the violent exothermal process. The energy barriers are from 60 to $80 \mathrm{~kJ} / \mathrm{mol}$, higher than the barriers of the formation of arenium ions. In other words, proton departure is the rate determining step, rather than the arenium ion formation. Besides, arenium ion is more stable than reactants. This means the kinetic and thermodynamic characteristics of this mechanism are quite different from the general mechanism of aromatic electrophilic substitution. 


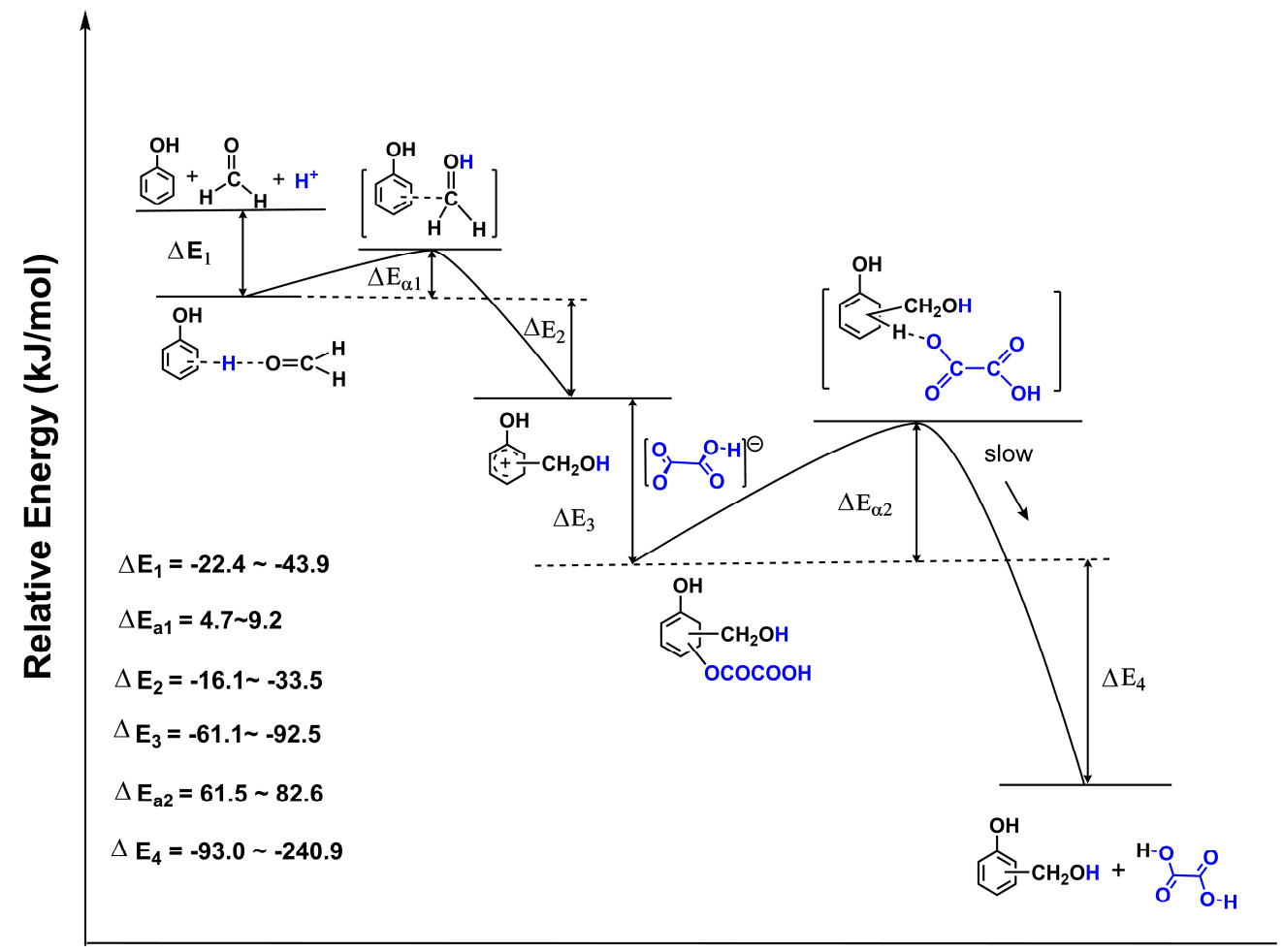

Figure 11. Energy profile of aromatic electrophilic substitution between phenol and formaldehyde with oxalic acid as catalyst.

\section{Conclusions}

Computational studies on aromatic electrophilic substitution between phenol and formaldehyde catalyzed by oxalic acid suggests an H-bond catalysis process. The calculation results of frontier orbitals show that the H-bond bridging interaction of substrate-catalyst complexes reduce the LUMO energy of formaldehyde and thus reduce the reaction barrier, thereby catalyze the reaction. Both the thermodynamic and kinetic characteristics based on this mechanism are different from those based on the generally accepted mechanism. H-bonded complexes are the attacking species of the substitution. The departure of proton, rather than the formation of arenium ion, is the rate determining step. Arenium ions are more stable than reactants. The whole reaction is highly exothermic, especially the step of departure of proton. The counterion of proton, oxalate anion, takes part in the reaction by bonding with arenium ion first, and then abstracting the proton to release the catalyst, oxalic 
acid. The H-bond catalytic mechanism perfectly explains the highly exothermic characteristic of the early stage reaction between phenol and aldehyde under acid catalysis. The essence of $\mathrm{H}$-bond catalysis is to lower the LUMO of the H-bond complexes of substrate-catalyst; as a result, the reaction barrier of the formation of arenium ion is very low.

\section{References}

(1) Zhang, Y.; Shen, S.; Liu, Y. The effect of titanium incorporation on the thermal stability of phenol-formaldehyde resin and its carbonization microstructure. Polym. Degrad. Stab. 2013, $98,514-518$.

(2) Chen, Y.; Hong, C.; Chen, P. The effects of zirconium diboride particles on the ablation performance of carbon-phenolic composites under an oxyacetylene flame. $R S C A d v . \mathbf{2 0 1 3}$, 3, 13734-13739.

(3) Knop, A.; Pilato, L. A. Phenolic Resins: Chemistry, Applications and Performance; Springer-Verlag, Berlin Heidelberg New York Tokyo, 1985.

(4) Peter, K.; Vollhardt, C. N.; Schore, E. Organic Chemistry: Structure and Function, 4th ed;

W. H. Freeman and Company, New York and Basingstoke, 2003.

(5) Astarloa-Alerbe, G.; Echeverria, J. M.; Egiburu, J. L.; Ormaetxea, M.; Mondragon,

I. Kinetics of phenolic resol resin formation by HPLC. Polymer 1999, 39, 3147-315 3.(6) Grenier-Loustalot, M. F.; Larroque, S.; Grenier, P.; Leca, J. P.; Bedel, D. Pheno lic resins: 1. Mechanisms and kinetics of phenol and of the first polycondensates tow ards formaldehyde in solution. Polymer 1994, 35, 3046-3054.

(7) Grenier-Loustalot, M. F.; Larroque, S.; Grande, D.; Grenier, P.; Bedel, D. Bedel P henolic Resins. 2. Influence of Catalyst Type on Reaction-Mechanisms and Kinetics. Polymer 1996, 37, 1363-1369.

(8) Xi, Y.; Hartwig, J. F. Hartwig. Mechanistic Studies of Copper-Catalyzed Asymmetric Hydroboration of Alkenes. J. Am. Chem. Soc. 2017, 139, 12758-12772

(9) Okumura, S.; Sun, F.; Ishida, N.; Murakami, M. Palladium-Catalyzed Intermolecular 
Exchange between C-C and C-Si $\sigma$-Bonds. J. Am. Chem. Soc. 2017, 139, 12414-12417.

(10) Hu, L.; Chen, H. Substrate-Dependent Two-State Reactivity in Iron-Catalyzed Alkene [2+2] Cycloaddition Reactions. J. Am. Chem. Soc. 2017, 139, 15564-15567.

(11) Ramos, M. J.; Fernandes, A. A. Computational Enzymatic Catalysis. Acc. Chem. Res. 2008, 41, 689-698.

(12) Cheng, G. J.; Zhang, X.; Chung, L. W.; Xu, L.; Wu, Y. D. Computational organic chemistry: bridging theory and experiment in establishing the mechanisms of chemical reactions. J. Am. Chem. Soc. 2015, 46, 1706-25.

(13) Tomberg, A.; Johansson, M. J.; Norrby, P.-O. A predictive tool for electrophilic aromatic substitutions using machine learning. J. Org. Chem. 2019, 84, 4695-4703.

(14) Oliveira, F. G.; Rodrigues, F. L.; de Oliveira, A. V. B. Marcal, D. V. L. M.; Esteves, P. M. Thoughts about the electrophilic aromatic substitution mechanism: the Friedel-crafts alkylation and acylation of benzene with acetyl and t-butyl catyions in the gas phase. Struct. Chem. 2017, 28:545-553.

(15) Liljenberg, M.; Stenlid, J. H.; Brinck, T. Mechanism and regioselectivity of electrophilic aromatic nitration in solution: the validity of the transition state approach. J. Mol. Model. 2018, 24: 15 .

(16) Galabov, B.; Nalbantova, D.; Schleyer, P. von R.; Schaefer, H. F. III. Electrophilic aromatic substitution: new insights into an old calss of reactions. Acc. Chem. Res. 2016, 49, 1191-1199.

(17) Liljenberg, M.; Stenlid, J. H.; Brinck, T. Theoretical investigation into rate-determining factors in electrophilic aromatic halogenation. J. phys. Chem. A 2018, 122, 3270-3279.

(18) Stuyver, T.; Danovich, D.; Proft, F. D.; Shaik, S. Electrophilic aromatic substitution reactions: mechanistic landscape, electrostatic and electric-field control of reaction rates, and mechanistic crossovers. J. Am. Chem. Soc. 2019, 141, 9719-9730.

(19) Conner, A. H. Predicting the reactivity of phenolic compounds with formaldehyde under basic conditions: An Ab Initio Study, J. App. Polym. Sci., 2000, 78, 355-363.

(20) Mitsunaga, T.; Conner, A. H.; Hill C. G. Jr. Reaction of formaldehyde with phenols: A 
computational chemistry study, Wood Adhesives, 2000, Session 2B: General, 147-153.

(21) Xu, W.F.; Xiong, S. S.; Shi, Y.Q.; Li, T. H.; Du, G. B.; Xie, X. G. Addition Mechanisms of Phenol toward Formaldehyde under Acidic Condition: a Theoretical Investigation. Chinese J. Struct. Chem. 2012, 31, 821-828.

(22) T. Li, M. Cao, J. Liang, X. Xie, G. Du, Theoretical Confirmation of the Quinone Methide Hypothesis for the Condensation Reactions in Phenol-Formaldehyde Resin Synthesis. Polymers 2017, 9, 45.

(23) Doyle, A. G.; Jacobsen, E. N. Small-Molecule H-Bond Donors in Asymmetric Catalysis. Chem. Rev. 2007, 107, 5713-5743.

(24) Vachal, P.; Jacobsen, E. N.Structure-based analysis and optimization of a highly enantioselective catalyst for the strecker reaction. J. Am. Chem. Soc. 2002, 124, $10012-10014$.

(25) Etter, M. C. Encoding and decoding hydrogen-bond patterns of organic compounds. Acc. Chem. Res. 1990, 23, 120-126.

(26) Akiyama, T.; Itoh, J.; Yokota, K.; Fuchibe, K. Enantioselective Mannich-Type Reaction Catalyzed by a Chiral Brønsted Acid. Angew. Chem. Int. Ed. 2004, 43, 1566-1568.

(27) Uraguchi, D.; Terada, M. Chiral Brønsted Acid-Catalyzed Direct Mannich Reactions via Electrophilic Activation. J. Am. Chem. Soc. 2004, 126, 5356-5357.

(28) Connon, S. J. Chiral phosphoric acids: Powerful organocatalysts for asymmetric addition reactions to imines. Angew. Chem. Int. Ed. 2006, 45, 3909-3912.

(29) Møller, C.; Plesset, M. S. Note on an Approximation Treatment for Many-Electron Systems. Phys. Rev. 1934, 46, 618-622.

(30) Gordon, M. H.; Gordon, T. H. Analytic MP2 frequencies without fifth-order storage. Theory and application to bifurcated hydrogen bonds in the water hexamer. Chem. Phys. Lett. 1994, 220, 122-128.

(31) Freeman, F.; Kasner, M. L.; Hehre, W. J. An ab initio molecular orbital theory study of 
the conformational free energies of 2-methyl-, 3-methyl-, and 4-methyltetrahydro-2 H-pyran. J. Mol. Struc.: THEOCHEM 2001, 574, 19-26.

(32) Kestutis, A.; Mikkelsen, K.V.; Stephan, P. A. On the Accuracy of Density Functional Theory to Predict Shifts in Nuclear Magnetic Resonance Shielding Constants due to Hydrogen Bonding. J. Chem. Theory Comput. 2008, 4, 267-277.

(33) Curtiss, A.; Redfern, P. C.; Raghavachari, K.; Rassolov, V.; Pople, J. A. Gaussian-3 theory using reduced Møller-Plesset order. J. Chem. Phys. 1999, 110, 4703-4709.

(34) Miertus, S.; Tomasi, J. Approximate evaluations of the electrostatic free energy and internal energy changes in solution processes. Chem. Phys. 1982, 65, 239-245.

(35) Tomasi, J.; Persico, M. Molecular Interactions in Solution: An Overview of Methods Based on Continuous Distributions of the Solvent. Chem. Rev. 1994, 94, 2027-2094.

(36) Glendening, E. D.; Reed, A. E.; Carpenter, J. E.; Weinhold, F. NBO Version 3.1, TC1, University of Wisconsin, Madison, 1998.

(37) M. J. Frisch, G. W. Trucks, H. B. Schlegel, G. E. Scuseria, M. A. Robb, J. R. Cheeseman, G. Scalmani, V. Barone, B. Mennucci, G. A. Petersson, H. Nakatsuji, M. Caricato, X. Li, H. P. Hratchian, A. F. Izmaylov, J. Bloino, G. Zheng, J. L. Sonnenberg, M. Hada, M. Ehara, K. Toyota, R. Fukuda, J. Hasegawa, M. Ishida, T. Nakajima, Y. Honda, O. Kitao, H. Nakai, T. Vreven, J. A. Montgomery, Jr., J. E. Peralta, F. Ogliaro, M. Bearpark, J. J. Heyd, E. Brothers, K. N. Kudin, V. N. Staroverov, T. Keith, R. Kobayashi, J. Normand, K. Raghavachari, A. Rendell, J. C. Burant, S. S. Iyengar, J. Tomasi, M. Cossi, N. Rega, J. M. Millam, M. Klene, J. E. Knox, J. B. Cross, V. Bakken, C. Adamo, J. Jaramillo, R. Gomperts, R. E. Stratmann, O. Yazyev, A. J. Austin, R. Cammi, C. Pomelli, J. W. Ochterski, R. L. Martin, K. Morokuma, V. G. Zakrzewski, G. A. Voth, P. Salvador, J. J. Dannenberg, S. Dapprich, A. D. Daniels, O. Farkas, J. B. Foresman, J. V. Ortiz, J. Cioslowski, D. J. Fox, Gaussian 09, Revision B.01, Gaussian, Inc., Wallingford CT, 2010.

(38) Schnatter, W. F. K.; Rogers, D. W.; Zavitsas, A. A. Teaching electrophilic aromatic substitution: enthalpies of hydrogenation of the rings of $\mathrm{C}_{6} \mathrm{H}_{5} \mathrm{X}$ predict relative reactivities; ${ }^{13}$ C NMR shifts predict directing effects of X. J. Chem. Educ. 2015, 92, 586-588. 
(37) Hiroko Sasaki.; Shigeyuki Daicho.; Yuji Yamada.; Yoshinori Nibu. Comparable Strength of $\mathrm{OH}-\mathrm{O}$ versus $\mathrm{OH}-\pi$ Hydrogen Bonds in Hydrogen-Bonded 2,3-Benzofuran Clusters with Water and Methanol. J. Phys. Chem. A 2013, 117, 3183-3189.

(38) Ridd, J. H. Diffusion Control and Pre-association in Nitrosation, Nitration and Halogenation. Adv. Phys. Org. Chem. 1978, 16, 1-49.

(39) Condon, F. E. Condon,Relationship Between Rates of Halogenation and Basicities of Methylbenzenes. J. Am. Chem. Soc. 1952, 74, 2528-2529.

(40) Olah, G. A.; Kuhn, S. J.; Flood, S. H. Aromatic Substitution. VII. Friedel-Crafts Type Nitration of Aromatics². J. Am. Chem. Soc. 1961, 83, 4564-4571.

(41) Olah, G. A.; Kuhn, S. J.; Flood, S. H.; Hardie, B. A. Aromatic Substitution. XIV. Ferric Chloride Catalyzed Bromination of Benzene and Alkylbenzenes wth Bromine in Nitromethane Solution. J. Am. Chem. Soc. 1964, 86, 1039-1044.

(42) Brown, H. C.; Brady, J. D. Solubility of Hydrogen Chloride at Low Temperatures. A Measure of the Basic Properties of Aromatic Nuclei; $\pi$ - and $\sigma$-Complexes and Their Role in Aromatic Substitution1,2. J. Am. Chem. Soc. 1952, 74, 3570-3582.

(43) Brown, H. C.; Stock, L. M. Relative Rates of Bromination of Benzene and the Methylbenzenes. Partial Rate Factors for the Bromination Reaction1-3. J. Am. Chem. Soc. 1957, 79, 1421-1425.

(44) Olah, G. A.; Uhn, S. J.; Flood, S. H.; Hardie, B. A. Aromatic Substitution. XXII: Acetylation of Benzene, Alkylbenzenes, and Halobenzenes with Methyloxocarbonium (Acetylium) Hexafluoro- and Hexachloroantimonate. J. Am. Chem. Soc. 1964, 86, 2203-2209. (45) El-Dusouqui, O. M. E.; Mahmud, K. A. M.; Sulfab, Y. ChemInform Abstract: $\pi$-Complex Formation and Collapse in Electrophilic Halogenation of Aromatic Substrates. Tetrahedron Lett. 1987, 28, 2417-2420.

(46) Sedaghat-Herati, M. R.; Sharifi, T. Substituent effects in aromatic substitution of aryltriethyltin compounds by mercuric halides. J. Organomet. Chem. 1989, 363, 39-44. 
(47) Walvoord R. R. Huynh P N H , Kozlowski M C . Quantification of Electrophilic Activation by Hydrogen-Bonding Organocatalysts. J. Am. Chem. Soc. 2014, 136, 16055-16065. 